\title{
Recursos virtuales en el aprendizaje de matemáticas en estudiantes de primaria en tiempos de covid-19
}

\author{
Cristabel Alvarez-Huillca \\ cristabelalvarez@hotmail.com \\ Juan Méndez Vergaray \\ jmvevaluaciones@hotmail.com \\ Johnny Félix Farfán-Pimentel \\ felix13200@hotmail.com \\ Dora Culqui-Culqui \\ doracc6@ hotmail.com \\ Edward Flores \\ eflores5000@gmail.com \\ Universidad César Vallejo \\ Lima - Perú
}

\section{RESUMEN}

La finalidad del presente artículo es comprender y reflexionar sobre las herramientas virtuales manejados en el proceso instruccional de la matemática en estudiantes de primaria en el contexto de la educación remota ocasionada por la COVID-19. En este espacio, los maestros han acudido a instrumentos de la tecnología actual para enfrentar el nuevo reto instruccional y de aprendizaje. La presente investigación exploró 104 artículos de las bibliotecas virtuales: Scopus, ESBCO y Scielo. Las evidencias de las pesquisas de los artículos examinados demuestran que las herramientas virtuales optimizan los aprendizajes en los estudiantes del nivel primario, tanto analógicos, no analógicos, como mixtos; las orientaciones al uso de los sistemas virtuales aportan estrategias amoldadas a la nueva realidad de la dualidad docente-aprendiz del nivel primario.

Palabras clave: aprendizaje; covid-19; estudiantes de primaria; herramientas virtuales. 


\title{
Virtual resources in the learning of mathematics in elementary school students in times of covid-19
}

\begin{abstract}
The purpose of this article is to understand and reflect on the virtual tools used in the instructional process of mathematics in elementary school students in the context of remote education brought about by COVID-19. In this space, teachers have resorted to current technology tools to face the new instructional and learning challenge. The present research explored 104 articles from the virtual libraries: Scopus, ESBCO and Scielo. The research evidence of the articles examined shows that virtual tools optimize learning in primary level students, both analogical, non-analogical and mixed; the orientations to the use of virtual systems provide strategies adapted to the new reality of the teacher-learner duality at the primary level.
\end{abstract}

Keywords: learning; covid -19; elementary students; virtual tools.

Artículo recibido: 02 noviembre. 2021 Aceptado para publicación: 28 noviembre 2021

Correspondencia: eflores5000@gmail.com Conflictos de Interés: Ninguna que declarar 


\section{INTRODUCCIÓN}

En el 2020, el inicio del azote de la COVID-19 en todo el orbe, alteró profundamente casi todas las actividades de la vida humana, asi también la educación, la cual generó cambios significativos en los seres humanos, ninguno estuvo exento de estas modificaciones; es por ello, en el sistema educativo, se realizó un replanteamiento sobre las responsabilidades de los educadores; por consiguiente, muchas instituciones adaptaron sus clases a una modalidad exclusivamente virtual; por lo tanto, en la actualidad, para continuar con este complejo proceso de transformación digital, se requiere docentes capacitados en educación a distancia: desde el dominio teórico-práctico de los recursos virtuales hasta un cambio en la metodología de la enseñanza (Lovón \& Cisneros, 2020).

Asimismo, Murillo \& Duk (2020) mencionan que lamentablemente son muy pocos los docentes que manejan con experticia los entornos virtuales; de esta manera, a nivel de diversos gobiernos de manera global implementaron recursos en línea y programas en medios de comunicación para su difusión, al respecto no todos los países de Latinoamérica tienen un plan que aproveche las TIC y las TAC de forma integral (CEPAL-UNESCO, 2020).

Debemos tomar en cuenta lo mencionado por Picón (2020) con respecto al aprendizaje en línea, presentándose como la más adecuada, debido al aislamiento que muchos gobiernos decretaron en casi todo el mundo, lo que representa una importante posibilidad para seguir con la enseñanza-aprendizaje en casa, de esta manera, evitar cualquier tipo de interrupción en la adquisición de nuevos conocimientos en las diferentes áreas.

Al mismo tiempo, Marciniak \& Gairín (2017) refieren, para que la enseñanza de manera virtual tenga éxito, debe mantener algunos criterios como tener la tecnología adecuada, poseer el servicio de internet para poder ingresar a la plataforma educativa entre otros aspectos. Asimismo, debe asegurar que los contenidos de los cursos en línea tengan un valor formativo con el cual se pueda lograr los aprendizajes esperados dentro del ambiente favorable para el docente y estudiante.

\section{Tipos de recursos virtuales}

En la actualidad se han implementado nuevos métodos de aprendizaje electrónico, insertándose nuevas formas de aprendizaje digital y a distancia, según la factibilidad y adecuado para dar garantía a la continuidad de la educación de los estudiantes en las 
instituciones educativas; es por ello, que el docente debe emplear metodologías completamente diferentes a las ya acostumbradas, siempre alineadas con el logro del aprendizaje del estudiante (Querevalú et al. 2021).

Por otra parte, de acuerdo con Chong \& Marcillo (2020), las plataformas virtuales otorgan contenidos en formatos diferentes: texto, sonido, video, hipertexto e hipermedia; mediante el cual se puede realizar la revisión, seguimiento y control de las actividades asignadas, actualmente, las utilizadas con mayor frecuencia se encuentra Moodle, Teams, Google Classrrom, Zoom, Google met, Jitsi meet, Canvas LMS, E-doceo y diversos sistemas de gestión de aprendizaje virtual. Es por ello que el docente elegirá aquellas que se adecúe a la enseñanza y estilo de aprendizaje de los estudiantes, así como a diversos factores económicos, institucionales y contextuales. Así también, Picón (2020) agrega que esto ocurre ante la urgente necesidad de que el docente pueda estar en contacto con sus estudiantes a través de las TIC y TAC para la interacción con sus estudiantes, es por ello que las instituciones públicas y privadas utilizan diversas estrategias con el propósito de lograr las competencias básicas en los educandos.

En Perú, las autoridades gubernamentales recurrieron a los recursos con los que se contaba para seguir las actividades con los alumnos, tales como WhatsApp, correo electrónico, YouTube, Moodle, Google Classrrom, Zoom, Jitsi, Meet, entre otros recursos. En tal sentido, que con el uso de los recursos virtuales, mencionadas por Molineros \& Chávez, (2019) los estudiantes vienen modernizando el modelo educativo, debido a que, a través de las TIC y las TAC, aprenden de manera diferente, ellos son considerados como nativos digitales y, desde pequeños, se apropian del funcionamiento de diferentes dispositivos; es asi, que esta situación genera que el comportamiento en clase sea diferente, pues esto es natural y cotidiano como tomar fotografías desde un celular y enviar las actividades a través del WhatsApp u otra aplicación.

\section{Herramientas virtuales usadas en las escuelas del Perú}

\section{- Moodle}

Guzzetti (2020) define al Moodle como un sistema informático para crear y compartir materiales educativos en línea. Moodle son las siglas de Modular Object-Oriented Dynamic Learning Environment, permite el aprendizaje en equipo, lo cual es sustancial para el proceso de socialización entre los estudiantes (Sánchez et al., 2012). 


\section{- Google Classrrom}

Pincay (2016) considera al Classrrom beneficioso para la educación ya que al ser una herramienta virtual para la enseñanza-aprendizaje que facilita y mejora las clases realizadas por el docente con el fin de lograr la comunicación y gestión de tareas, esto permite que los estudiantes organicen sus trabajos y compartir en línea con el uso de la herramienta Google Drive, en consecuencia, esta herramienta virtual se adapta perfectamente al trabajo grupal, de tal forma que el maestro tiene la oportunidad de crear un aula de clase con un grupo de estudiantes, siendo él quien se convierte en moderador, pudiendo asignar actividades de manera segura.

\section{- Zoom}

Para el uso del zoom, Ayala (2021) comparte la manera de trabajarlo con los estudiantes y hace uso de las herramientas que la misma plataforma proporciona. Él menciona que esta presentación consiste en una sesión de clase que empieza con un breve saludo, para acceder a la sala, se puede configurar un filtro de ingreso o dejarlo sin restricción, enseguida es posible compartir la pantalla para mostrar una noticia reciente o brindar instrucciones para que se marque cierta imagen, se coloque timbres o se realice anotaciones, después si el caso lo amerita, se puede silenciar el micrófono de los participantes para evitar el ruido o la intervención de terceros no autorizados, para intervenir en la clase, se cuenta con la herramienta "levantar la mano", de esta manera, se puede comunicar el deseo de intervenir, luego, se podría dar instrucciones para responder un quiz o, también, ir a la secuencia de diapositivas; además, se puede subdividir en grupos más pequeños en la cual el docente realiza el monitoreo del trabajo en equipo.

\section{- Google Meet}

El Google Meet es otra aplicación muy utilizada debido a su gratuidad, esta es la reciente plataforma virtual de Google que reemplaza a su antecesora Hangouts, aquí el estudiante ingresa mediante una contraseña que se crea cada vez que se utiliza para la conferencia, dando mayor seguridad y es posible programar clases posteriores con anticipación (Chong \& Marcillo, 2020). Es por ello que, las Instituciones educativas que utilizan Google Meet, según lo mencionan Cedeño-Escobar et al. (2020), graban sus clases para que el estudiante las pueda visualizar en el momento que requiera repasar el tema o no haya podido ingresar. 


\section{- WhatsApp}

El acceso a plataformas virtuales depende de los megas que tenga el padre de familia; es por ello, que se evidenció que la aplicación más utilizada para las clases virtuales es el WhatsApp, esto debido a su fácil manejo y acceso; asimismo, es importante mencionar que el docente, a través de este aplicativo, puede generar grupos para que los padres descarguen el material de trabajo que incluye imágenes, audios, archivos y otros materiales; de esta manera, se logra comunicar, de forma oportuna, los avances de los estudiantes quienes, a través de esta herramienta, suben sus actividades de forma diaria (Crespo \& Palaguachi, 2020). Además, debido a la inmediatez de este servicio de mensajería, las tareas y asignaciones presentadas pueden revisarse y actualizarse rápidamente. En la misma línea, Padrón (2013) detalla que el WhatsApp se posiciona como una de las aplicaciones para móviles más utilizada debido a su forma de comunicación en tiempo real.

\section{La virtualidad en el aprendizaje en Perú}

El Gobierno peruano consideró necesario interrumpir las actividades escolares presenciales a partir del 16 de marzo de 2020. Para dicho fin, se emitió la Resolución Viceministerial N. ${ }^{\circ}$ 079-2020 (MINEDU, 2020). En plena emergencia sanitaria y en

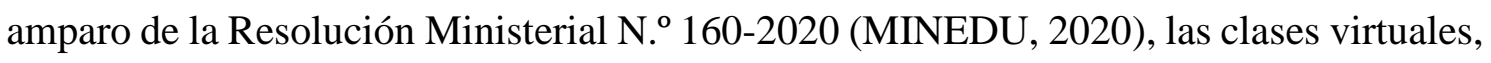
en centros educativos estatales, se iniciaron el 6 de abril de ese mismo año bajo el programa de educativo virtual "Aprendo en Casa". El nuevo contexto para los profesores, estudiantes y padres de familia imponiéndose retos e interrogantes referentes a la preparación de los docentes en herramientas virtuales (Campos et al., 2021).

\section{Aprendo en casa}

En el contexto actual que se atraviesa se han ideado un conjunto de propuestas educativas que tienen el propósito de favorecer el proceso educativo de los estudiantes en los distintos niveles educativos, sustancialmente en la educación básica con miras a dar énfasis a los aprendizajes y lograr el desarrollo de capacidades fundamentales, es por ello que la estrategia Aprendo en casa, se presentó como una oportunidad para brindar soporte pedagógico tanto a estudiantes como a docentes, esto generó una buena expectativa en la comunidad educativa, brindándose de manera multicanal como la internet, la televisión y la radio ofreciendo experiencias de aprendizajes novedosas y buscando motivar a la población educativa a un mayor interés en su proceso formativo integral. 
Aprendo en casa, como propuesta pedagógica brinda un conjunto de posibilidades a los educandos con el firme propósito de contribuir al desarrollo de capacidades y habilidades cognitivas, la estrategia en mención se encuentra organizada en un conjunto de actividades que potencian los aprendizajes y materiales en formato digital, estas se encuentran a disposición del personal docente y estudiantes para efectivizar el desarrollo interactivo y colaborativo de la clase; en su diseño se cuenta con un planificador de sesiones de aprendizaje, fichas de trabajo didáctico, videos introductorios y recursos digitales de fácil acceso; así también se encuentran habilidades a través de plataformas digitales y de redes sociales (Morales, 2020).

\section{Aprendizajes en la virtualidad}

A raíz de cambiar el enfoque en la entrega de información a las oportunidades de comunicación en los esquemas de los sistemas de gestión del aprendizaje, la aplicación de contenido digital está recibiendo más atención que el contenido humano, mientras se crean entornos de aprendizaje en red. Por consiguiente, la tendencia es dar la importancia a la práctica de la educación promoviendo la tolerancia, cooperación y reflexión en los campos del aprendizaje en red o en línea (Cabrera-Berrezueta, 2020).

La relación establecida entre la institución educativa y la sociedad es el soporte fundamental del proceso educativo al introducir las TIC y TAC como parte adicional para seguir adquiriendo conocimientos, el empoderamiento en actitudes valorativas y desarrollando estrategias adecuadas para aprender en concordancia con las falencias personales y grupales. Empero, el desastre de sanidad en el ámbito global ha obligado a asumir medidas urgentes de asociación interdisciplinar y transversal con el apoyo tecnológico; de suerte que la información esté en condiciones de seguir una secuencia de procedimientos facilitadores de la acción pedagógica en la que la dualidad maestroaprendiz se encuentren en condiciones de alcanzar las metas del proceso educativo, teniendo como pilar el aprendizaje significativo e inmediatamente adaptarse a esta nueva realidad con una actitud solidaria, colaborativa y participativa de la trilogía maestroaprendiz-contexto (Silva et al., 2020).

Asimismo, en el aprendizaje, como lo menciona Quirós (2009), la incorporación de las TIC y las TAC marcan pautas eficaces en los constantes cambios de los modelos educativos, es por ello, que los docentes deben capacitarse de manera constante en recursos virtuales lo cual permitirá a los estudiantes recibir una educación de calidad con 
estrategias innovadoras para lograr que el estudiante cambie su conducta y el desarrollo de diversas habilidades. Para García et al. (2015), es importante conocer cuáles son las actividades que permiten que el estudiante logre el aprendizaje, esto permitirá que el docente emplee diversas estrategias para lograr el aprendizaje esperado. o, más aún, en la aplicación que se realice de dicha información por parte de los estudiantes.

\section{Teorías del aprendizaje}

\section{- Teoría conductista}

Se basa en el empleo de procedimientos, los cuales permiten estudiar el aprendizaje con los cambios en la conducta que se puedan observar, cuyo fin es que, al dar el estímulo, sea posible predecir cuál será la forma de responder, y dada la respuesta estar en condiciones de pronosticar el estímulo. Es importante entender que la corriente conductista indaga las conductas y comportamientos, siendo los estados mentales materia de otras disciplinas científicas, es por ello, los conductistas afirman que el aprendizaje genera un cambio de conducta (Ertmer \& Timothy, 1993). Además, para Silva-Sánchez et al. (2020), en el conductismo, el conocimiento mediante una computadora debe ser organizado de manera secuencial, debe evaluar la observación conductual en concordancia con las metas propuestas; así mismo, el proceso evaluativo debe tener en consideración los contenidos que se desarrollen para poder arribar al logro de los objetivos establecidos.

\section{- Teoría cognoscitivista}

Para Silva-Sánchez et al. (2020) agrega que el cognoscitivismo, en la era virtual, el usuario es el que procesa la información invitando al estudiante a combinar la información con el mundo real brindando información propia debido al conocimiento adquirido.

\section{- Teoría Constructivista}

En el constructivismo, el aprender implica la consolidación de la interrelación entre los aprendices y aprendiz-acompañante, de acuerdo como se vayan construyendo nuevas estructuras mentales, se desarrollen nuevos conocimientos, se acepten nuevas tecnologías que contribuyan con los aprendizajes como el Internet y su extensa gama del saber; sin embargo, es imprescindible que la experticia del maestro debe hacerse presente para dar orientaciones al aprendiz en la selección de la información pertinente y congruente con la metodología, la ética y valores que deben estar presente en el proceso de aprendizaje (Silva et al., 2020). Asimismo, en la virtualidad, se presentan guías para aprender, que se 
hacen evidente con mayor frecuencia desde que se implementó la Web 2.0 como herramienta comunicativa en el ámbito tecnológico, esto ha permitido interactuar al aprendiz con los contenidos y diferentes cibernautas en espacio-tiempo real; lo que dado como consecuencia al incremento de la información colaborativa para facilitar el análisis y asumir conclusiones personales para empoderarse en nuevas opiniones y nociones facilitadas por la transversalidad (Silva et al., 2020).

\section{- Conectivismo}

Es la teoría del aprender, que se centra en el ámbito digital; es decir, los aprendizajes son posibles de realizarse a partir de la conectividad en la red; asimismo, se encuentra orientada en generar interacciones conectistas gestionando conjunto de datos organizados que posibilitan poseer un mayor soporte informacional y que es el sustrato para la generación de nuevos conocimientos (Siemens, 2004). Es por ello que, el conectivismo se presenta un correlato de la competitividad del trabajo; en el que trabajador se ve obligado a estar permanentemente actualizado y adquiriendo nuevos conocimientos en línea o teletrabajo en contextos no formales (Silva-Sánchez et al. 2020).

\section{Recursos virtuales y aprendizaje de las matemáticas}

Los docentes del área de matemática, deben saber la importancia que tiene su curso para la preparación de estudiante en todos los aspectos que involucra el aprendizaje. Asimismo, el docente debe sentir gusto por lo que realiza e ir integrando a sus actividades los recursos virtuales para que las clases no se tornen monótonas y aburridas, lo que ocasionaría que no se logre el aprendizaje esperado (Muñoz-Vázquez et al. 2020). Por lo tanto, el reto que debe afrontar el docente es el uso de las herramientas tecnológicas para poder enseñarles a los estudiantes como usarlas de manera adecuada en favor de la adquisición de nuevos conocimientos (Sánchez, 2020). En ese sentido, muchos docentes mantienen arraigada la enseñanza tradicional, reflejándose en el uso de la tecnología de manera reducida debido a que aún mantienen este tipo de concepto. Por lo cual se evidencia a muy pocos docentes que utilizan las TIC y las TAC. Es por ello la importancia de manejar los recursos virtuales para lograr aprendizajes en los estudiantes, esto permitirá al docente seleccionar de forma adecuada las actividades a implementar, considerando una revisión previa de las mismas en torno a su funcionamiento, claridad y comprensión (Muñoz-Vázquez et al., 2020). 


\section{Herramienta virtual usada para el área de Matemática}

\section{Kahoot}

Esta herramienta solo necesita una conexión a Internet, que se puede obtener a través de cualquier dispositivo fijo o móvil. Además, Kahoot no necesita software, conocimientos o gestión previos, mientras que las preguntas se pueden crear en muy poco tiempo y de forma sencilla. Además, permite utilizar las respuestas de los alumnos ya que no hay un número limitado de comandos a distancia. Por otro lado, este dispositivo da la oportunidad de construir ítems de elección variada, con la cantidad requerida e incluyendo iconografía, videos y esquemas que permiten una presentación más interesante para los aprendices (Valles-Pereira \& Mota-Villegas, 2020). Además, Kahoot se encuentra en la web a disposición de los usuarios sin ningún costo; es utilizada para elaborar situaciones lúdicas interactivas que tienen como base interrogantes con la participación de parejas o cualquier cantidad de participantes en forma paralela (Martínez, 2017). Para el uso del Kahoot el maestro deberá elaborar un banco de preguntas con imágenes u otros si así lo requiere. Asimismo, determinar el tiempo que durará cada pregunta y las opciones ,el docente y los estudiantes podrán visualizar el puntaje obtenido (Valles-Pereira \& MotaVillegas, 2020).

\section{Objetivo}

- Verificar los recursos virtuales utilizados con mayor frecuencia por estudiantes del nivel primario en las Instituciones educativas.

- Analizar cómo estos recursos virtuales, tienen influencia en el desarrollo de la educación.

- Exponer las herramientas más destacadas para que los docentes puedan desarrollar y emplear metodologías heterogéneas en el aula y propiciar el mejoramiento del rendimiento de los alumnos.

- Precisar los recursos virtuales para la actualización permanente del docente.

\section{ESTRATEGIAS METODOLÓGICAS O MATERIALES Y MÉTODOS}

El método utilizado en esta pesquisa es el analítico, debido a que se describió el contexto de forma general, este conocimiento nos permite conocer y explicar las características de cada uno de los elementos básicos y las relaciones. Asimismo, el avance tecnológico encontrado permitirá cuestionarnos acerca de cuáles son los recursos que más utilizan los docentes para lograr el aprendizaje en los estudiantes; además, verificar si éstas suponen 
progresos en forma como se perciben los contenidos vertidos en la clase, así como que tan efectiva se muestra en la ejecución de los procesos de los aprendizajes en relación a la enseñanza,; teniendo en cuenta determinados indicadores que precisen cuán efectiva es la labor educativa (Molinero \& Chávez, 2019).

La pesquisa se ejecutó entre los meses de julio y agosto del 2021; los artículos fueron ubicados en la base de datos Scopus, Scielo y Ebsco. Para la búsqueda se utilizó las ecuaciones: Recursos virtuales AND Aprendizaje AND Estudiantes de primaria, Herramientas educativas AND Pandemia Virtual Resources AND Learning AND Primary school students . Además, se realizó búsqueda adicional con el fin de tocar el tema de recursos virtuales en el aprendizaje de la matemática.

Se consideró los criterios de exclusión en artículos que se alejaban del tema investigado ya que no eran de interés para la investigación. Dentro de nuestro discernimiento de inclusión se tomó en cuenta los títulos, resumen, metodología y resultados; tomando en cuenta los documentos que más se acercaban al objetivo, seleccionándolos para ser descargados en PDF y registrarlos en el gestor Mendeley web para que el desarrollo de nuestra investigación se vea ordenada, teniendo en cuenta el formato APA colocando las referencias bibliográficas de manera automática en el documento. Fueron encontrados 104 investigaciones en la base de datos antes mencionadas, de los cuales 30 fueron excluidas por ser repetitivas, quedando 73 para ser analizadas en la investigación.

\section{RESULTADOS Y DISCUSIÓN}

\section{Resultados}

$\mathrm{Al}$ analizar variedad de artículos relacionados con los recursos virtuales en la enseñanza de la matemática en alumnos de primaria durante la pandemia provocada por la COVID19, se encontró la relevancia del uso de las nuevas estrategias y recursos que mejoran el proceso de aprendizaje en esta nueva realidad. Es por ello que el aula virtual favorece los aprendizajes de los estudiantes potenciando sus capacidades humanas, en este ambiente de trabajo pedagógico se desarrolla el proceso interactivo con los estudiantes buscando permanentemente que los estudiantes encuentren la motivación tan necesaria, utilizando una gama de recursos y materiales didácticos, asi también ofreciendo todas las posibilidades que el entorno virtual lo posibilite (Kraus et al.2019).

En ese sentido, de la revisión de diversos estudios científicos sobre el tema en mención se obtuvieron los resultados tal como en la investigación realizada por Ligon (2021), se 
evidenciaron resultados que permitieron a los maestros crear objetivos de aprendizaje diarios más manejables, utilizando herramientas Web 2.0 que eran más apropiadas para sus lecciones, estos cambios en la planificación e instrucción de las lecciones ayudaron a aumentar la participación de los estudiantes. Por otra parte, los estudiantes pudieron comprender mejor y aplicar lo que estaban aprendiendo, a partir de estas prácticas se evidenció que la participación y el compromiso aumentaron a medida que los maestros encontraron nuevas formas de hacer que sus lecciones fueran más inclusivas y las interrupciones de los estudiantes disminuyeron a medida que esto continuaba sucediendo. Al mismo tiempo, la investigación de Ramitadila et al. (2020) muestran que, para diseñar el aprendizaje en línea con diversas estrategias de aprendizaje en armonía con un plan de estudios nacional, es fundamental la preparación tecnológica y la colaboración para alcanzar el éxito del aprendizaje en línea.

Por otro lado, Marbán et al.(2021) enfatizan en sus investigaciones que el uso del aprendizaje digital en matemáticas brinda a los estudiantes una oportunidad significativa para estudiar y completar su aprendizaje desde sus hogares en tiempos de crisis, obteniendo como resultados que no existen diferencias significativas en la implementación de plataformas de redes sociales durante el aprendizaje de las matemáticas en función del género, los grupos de edad y el tipo de escuela, así como el uso de aplicaciones de redes sociales para el aprendizaje matemático depende del nivel educativo de los padres y del nivel económico de la familia. Concluyó que los estudiantes no estaban familiarizados con el uso de la plataforma de redes tecnológicas en su aprendizaje.

Rybak (2021) describe la efectividad del uso de las TIC en la escuela secundaria, se trató de un estudio preliminar de lo que sucedía en la escuela, en el aula, en el trabajo didáctico cotidiano con el uso de las TIC en situaciones didácticas adecuadas y en las circunstancias escolares normales. El resultado fue el progreso en el uso de la computadora para cambiar el estilo de trabajo durante la lección de matemáticas (desde dibujar gráficos en un cuaderno hasta concluir y estructurar el conocimiento) el cual fue visible y bastante rápido. Es por ello que, algunos estudiantes notaron mejores resultados en sus pruebas de clase después de las lecciones con la computadora. Tal vez sea el resultado de una mejor comprensión de los temas o la posibilidad de resolver más ejercicios en lugar de dibujar gráficos a mano. 
Con respecto al impacto en el entorno de aprendizaje virtual, según Martín et al.(2021) los resultados obtenidos son los siguientes: el hecho de conocer el uso, la creación y la distribución de los recursos tecnológicos por parte del profesorado para que estén en concordancia con los objetivos, los estudiantes y el estilo de enseñanza es vital. Por otro lado, los estudiantes creen que los profesores carecen de los conocimientos necesarios para utilizar editores de imágenes, editores de video, editores de gráficos por computadora. Asimismo, es de suma importancia encontrar los factores que impiden que los estudiantes alcancen un nivel óptimo en el tratamiento de estas herramientas durante el transcurso de sus estudios, lo que les permitirá adoptar otra serie de técnicas y/o decisiones.

Es importante observar los desafíos que se enfrenta en el modo en línea se minimiza la enseñanza, al igual que los problemas de red, minimizados debido a la conveniencia y disponibilidad de la red; como un alumno puede ver videos de acuerdo con su ritmo, la participación del alumno aumenta automáticamente. Sin embargo, los estudiantes están satisfechos con este tipo de aprendizaje, ya que les da tiempo para aprender en cualquier lugar y en cualquier momento con su propio dispositivo y ritmo, más del $83 \%$ de los estudiantes estaban contentos con este modo de aprendizaje; el maestro también puede monitorear la participación del estudiante a través de la evaluación de cuestionarios, tareas y actividades que ilumina el aprendizaje autodirigido y el aprendizaje participativo, es por ello que, el profesor también está satisfecho con este modo, aunque lleva tiempo preparar el material de aprendizaje (Pratibha et al., 2021).

Desde el punto de vista de Velychko et al. (2021) quienes analizan el uso eficaz de los recursos educativos electrónicos abiertos, dando como resultado el proporcionar una educación integral, equitativa e incentivar mejoras, son prioridades del desarrollo sostenible de la sociedad, es pues que esta forma de educación se realiza mediante la implementación de las ideas de educación abierta, en la que los recursos educativos electrónicos abiertos juegan un papel crucial, el desarrollo de recursos educativos electrónicos es un proceso responsable y costoso; en consecuencia la implementación del desarrollo de recursos educativos electrónicos de calidad solo es posible en cooperación con especialistas en diversas especialidades.

En su estudio, Francisca et al. (2021) analizaron la percepción de los estudiantes sobre las plataformas educativas ecuatorianas en la educación durante la pandemia COVID- 
19. El estudio fue cuantitativo. La muestra fue participativa no probabilística, conformada por estudiantes de diferentes instituciones de la Provincia de Chimborazo. El resultado fue que existe una percepción positiva en los estudiantes que utilizan las plataformas educativas. Hubo diferencias estadísticas significativas según las variables de género, contrario a los niveles de estudio y ubicación. Existe un mayor afecto solidario de los estudiantes al recibir clases virtuales de las profesoras, concluyendo que la pandemia COVID-19 aceleró la adopción del aprendizaje en línea por parte de las instituciones.

El entorno de aprendizaje interactivo es una de las claves del aprendizaje exitoso; uno de los cuales es provocado por una selección efectiva de tecnología implementada. Latif \& Widiaty, (2021) tenían como objetivo investigar la implementación de tecnología promoviendo el aprendizaje digital, identificando una variedad de plataformas digitales e ir descubriendo que plataformas como el entorno virtual, los juegos digitales, las plataformas de aprendizaje basadas en la web, los laboratorios / simulaciones virtuales, los dispositivos móviles, los robots y las redes sociales son tecnologías eficaces para promover el aprendizaje digital.

Por su parte, Hidayah \& Prayoga( 2021) mencionan que la actitud de los aprendices entorno a las matemáticas con los indicadores de formación del aprendizaje por descubrimiento con manipulativos concretos y virtuales es bueno; así también las actitudes de los aprendices hacia las matemáticas en el aprendizaje por descubrimiento utilizando manipulativos concretos es mayor que el aprendizaje por descubrimiento utilizando manipuladores virtuales; como también los indicadores de actitud tienen un alto efecto hacia las matemáticas de los estudiantes en el aprendizaje por descubrimiento con manipulativos concretos y virtuales en el mismo orden. Asimismo, Corrales ( 2021) al estudiar la asociación entre la forma de enseñar las matemáticas y los entornos virtuales para aprender, halló que la mayoría de los colaboradores consideraban que los EVA como la TIC se constituían en facilitadores para aprender significativamente los contenidos de matemática, así como para incrementar la motivación y los procesos metacognitivos.

Para A. M. Martín et al.(2021) el papel de la coordinación del Consorcio en este proyecto ha sido fundamental para desarrollar un cambio de modalidad educativa y fortalecer las competencias tecnológicas y pedagógicas; tal es así que esto redundará en una mayor eficiencia y mejor desarrollo de la modalidad de educación a distancia de calidad en un grupo de instituciones que se encuentran en la etapa denominada básica y/o inicial en el 
proceso de transición a una metodología virtual / en línea, es decir, en el marco de una educación virtual de emergencia.

Por otro lado Lin et al. (2010) mencionan que los resultados del estudio proporcionan fuertes evidencias empíricas sobre los comportamientos de aprendizaje y los patrones de interacción de los estudiantes dentro de los mundos virtuales de aprendizaje, que es una de las herramientas más destacadas en la educación en la actualidad, el alcance de los impactos del estudio en la práctica de los mundos virtuales de aprendizaje es limitado. por la falta de medición relacionada con el rendimiento del aprendizaje en el diseño de la investigación. Sin embargo para Prieto et al.( 2019) los resultados obtenidos, tras la valoración, han sido muy positivos. Globalmente, los estudiantes consideran que han podido autoevaluar su proceso de aprendizaje, el cual ha sido más activo y vivencial y han tenido la posibilidad de mostrar mejor lo aprendido, lo cual resulta atrayente desde desde la perspectiva del proceso metacognitivo, podemos decir que la integración de dispositivos móviles en el aula, como el teléfono inteligente, que utilizan los estudiantes en el día a día, favorece el proceso de enseñanza-aprendizaje. De esta forma, se crea un ambiente en el aula basado en la motivación y mejora en la adquisición de nuevos contenidos, que incluye siempre un sistema de control adecuado para el docente y las características del grupo-clase.

En la investigación realizada por Carpenter \& Joshua, (2020) mediante el cual se examinó las experiencias educativas de las familias en el marco del COVID-19 y sus decisiones de escolarización en el curso 2021. Los resultados de una encuesta realizada a 1.743 padres indican que la mayoría de las escuelas proporcionaron recursos educativos que van desde paquetes impresos hasta instrucción en línea en vivo. En general, los padres valoraron positivamente la experiencia. Los padres de los colegios privados y concertados informaron de una experiencia más positiva que los de los colegios públicos tradicionales. Sólo un pequeño porcentaje de los encuestados dijo que iba a educar en casa, pero más de un tercio planeó enviar a su hijo a una escuela virtual por la preocupación por la salud de su hijo.

Ardini et al.(2020) en su estudio para el logro de sus objetivos con respecto a herramientas digitales comunicativas contextualizado en la COVID-19, aplicó un cuestionario con características aleatorias a 194 participantes en la ciudad de Córdoba, Argentina. Asimismo, se recogieron datos lográndose evidenciar mejoras en los aprendizajes, así 
como dificultades a superar; en suma, la continuidad de situaciones problemáticas del contexto socioeducativo preexistentes se profundizó a raíz de la pandemia dando origen a nuevos cuestionamientos idear propuestas de una educación cuyo compromiso pleno para el desarrollo de los ciudadanos.

Por otro lado Rybak ( 2021) en esta investigación, se evaluaron el aprendizaje basado en proyectos identificando la teoría y la resolución de problemas con mapas mentales virtuales; y los productos de aprendizaje en forma de audiovisuales y la comunicación de los resultados en forma de carteles virtuales podrían aplicarse para observar el continuo del pensamiento visual de los estudiantes. Se utilizan mapas mentales virtuales para observar el pensamiento visual, audiovisuales para observar el aprendizaje visual y carteles virtuales para observar la comunicación visual de los estudiantes.

Wahyuningsih et al.(2021)mencionan como resultado de su investigación, evaluando el aprendizaje basado en proyectos identificando la teoría y la resolución de problemas con mapas mentales virtuales; siendo los productos de aprendizaje en forma de audiovisuales y la comunicación de los resultados en forma de carteles virtuales podrían aplicarse para observar el continuo del pensamiento visual de los estudiantes; también se utilizaron mapas mentales virtuales para observar el pensamiento visual, audiovisuales para observar el aprendizaje visual y carteles virtuales para observar la comunicación visual de los estudiantes.

Es necesario tomar en cuenta lo mencionado por Addimando et al. (2021) al presentar un caso en el que se implementaron tecnologías digitales para la educación en línea en particular, la educación matemática en la escuela primaria italiana. Los resultados confirman que la rápida transición a la forma de educación en línea, debido a la pandemia COVID-19, tuvo éxito y aportó experiencia que puede ser útil en futuras actividades didácticas.

\section{Discusión}

La actual crisis de salud ha provocado una interrupción sin precedentes en la educación de los estudiantes de las escuelas en todos los niveles. Para mantener la continuidad de la educación de los educandos durante el tiempo de emergencia, todo el sistema de educación ha cambiado por primera vez a un modo de educación en línea utilizando diferentes plataformas digitales. 
Por lo tanto, el conocer las herramientas virtuales no es tarea fácil, en lo académico, entre las percepciones positivas del uso de recursos virtuales se destacan la flexibilidad del tiempo, la claridad y los recursos de apoyo para realizarlas. Una de las dificultades que se evidencian es la sobrecarga de trabajos, en cuanto al aprendizaje, la atenciónconcentración y la dificultad para resolver las actividades, y la falta de socialización en entornos presenciales es lo que más extrañan los estudiantes (Sosa, 2021). Por otro lado, es importante mencionar que los estudiantes fueron los verdaderos héroes porque se les pidió mucho esfuerzo; así también cuando se hizo que el aprendizaje fuera accesible, atractivo y valioso para ellos, estuvieron dispuestos a dar un paso adelante para aprender y mostrarnos lo que aprendieron; es por ello que, durante este curso escolar de COVID19, el aprendizaje virtual puso de manifiesto nuestras debilidades y mostró la falta de consideración para proteger a algunas de nuestras poblaciones más vulnerables (Ligon, 2021).

Asimismo, en pocos meses, los sistemas educativos nacionales recurrieron a soluciones como los métodos en línea utilizando aplicaciones en línea, televisión, radio y métodos fuera de línea, incluyendo libros y módulos impresos. Junto con estos cambios, ha sido necesario modificar las estrategias de instrucción, la preparación tecnológica para implementar el aprendizaje en línea y proporcionar apoyo y motivación a todas las partes interesadas (Ramitadila et al., 2020); en este sentido la mediación de la tecnologías en los entornos virtuales necesita que el aprendiz sea capaz de construir y reconstruir sus habilidades y destrezas cognitivas fundamentales en función de su experticia, intereses, perspectivas y sapiencia para elaborar su aprendizaje personal, utilizando métodos propios y con disciplina (Guzzetti 2020).

Ahora bien, el mejor método para la enseñanza de las matemáticas a distancia en la época de la COVID-19 es seguir el plan de estudios de matemáticas existente, centrándose en la profundidad del contenido, aplicándolo a un ritmo más lento y con un contenido reducido. Además del libro del plan de estudios de matemáticas, los materiales de autoaprendizaje deben ser diseñados y luego subirlos a la página web oficial del Ministerio de Educación, donde están disponibles para todos los estudiantes Marbán et al.( 2021), en este sentido los materiales deben incluir una serie de instrucciones relacionadas con el aprendizaje de la habilidad y enlaces a contenidos digitales de apoyo ; un vídeo educativo, un clip de audio, un juego educativo y otros. 
Las TIC son un importante recurso didáctico en la escuela moderna. Cada nueva herramienta didáctica se introduce en el proceso educativo con el fin de aumentar la eficacia de este proceso, debido a que se busca que los estudiantes puedan utilizar la herramienta virtual más eficiente Rybak, ( 2021),es por ello se evidencia una mayor simpatía de los estudiantes al recibir clases virtuales por parte de los docentes ,concluyendo que la pandemia de COVID-19 aceleró la adopción del aprendizaje en línea por parte de las instituciones de educación (Martínez, 2020).

El proceso de enseñanza-aprendizaje ha evolucionado enormemente en los últimos 5-10 años. El modo de enseñanza ha cambiado de la pizarra normal a la enseñanza tecnológica. Debido al brote de COVID-19, el sistema educativo se ha visto perturbado especialmente; afecta más a las Instituciones educativas. La mayor parte del sector educativo se ha pasado a la enseñanza a distancia Pratibha et al.( 2021),es por ello que el docente se está habituando a utilizar los recursos tecnológicos y muestra una ligera satisfacción, aunque le lleve tiempo preparar el material didáctico.

Proporcionar una educación integral y equitativa de calidad y promover las oportunidades de aprendizaje permanente para todos son prioridades del desarrollo sostenible de la sociedad, esta forma de educación se realiza mediante la aplicación de las ideas de la educación abierta, en la que los recursos educativos electrónicos abiertos desempeñan un papel crucial. Sin embargo implementar recursos educativos electrónicos resulta costoso, es por ello que solo será posible en cooperación con especialistas de diversas especialidades y el gobierno (Velychko et al., 2021).

Las plataformas educativas son un medio importante para desarrollar las clases virtuales. Los estudiantes desarrollan habilidades y metacogniciones que les permiten aprender de forma autónoma y autorregulada, estas herramientas facilitan la comunicación, establecen un acercamiento entre el profesorado y el alumnado, la cual puede ser sincrónica y asincrónica Francisca et al.(2021).Asimismo, se puede concluir que el uso de la tecnología ayuda a aumentar la motivación y el aprendizaje de los estudiantes, estimula la innovación y la creatividad, aumenta la capacidad de pensar y resolver problemas, es capaz de aumentar la interacción y la comunicación en grupos y la cooperación (Latif \& Widiaty, 2021). 
Asimismo, la totalidad de docentes en formación o en servicio reconoce los beneficios del uso de las TIC y las TAC para la educación matemática, teniendo en consideración e insertar en los planes de mejora y capacitación de docentes (Corrales, 2021).

La característica más notable fue que las instituciones educativas no estaban preparadas metodológicamente para el cambio, todas tenían la característica común de no tener un modelo educativo diseñado para un formato virtual/online. La mayor necesidad que han mostrado es el fortalecimiento metodológico y las competencias pedagógicas para ofrecer un aprendizaje virtual significativo A. M. Martín et al.( 2021), sin embargo la falta de conectividad era una realidad, así como la brecha digital existente en los diversos lugares del Perú es por ello que los planes de formación para los estudiantes y los docentes han sido una estrategia clave en el proceso.

Los mundos virtuales de aprendizaje podrían crear un entorno de aprendizaje en red que no sólo se asemeje a los entornos de aprendizaje de la vida real, sino que también aumente el valor de la educación tradicional al sumergir a los estudiantes en el espacio de aprendizaje virtual y ampliar el horizonte de las experiencias de aprendizaje, en el sentido de que los mundos virtuales de aprendizaje podrían ser muy prometedores para resolver los problemas de las comunidades virtuales de aprendizaje y los apoyos al aprendizaje en entornos de aprendizaje en red (Lin et al., 2010), para mejorar la práctica de los aprendizajes virtuales es importante explorar e investigar cómo los estudiantes se comportan dentro de los mundos virtuales de aprendizaje y en la comunicación con sus compañeros.

La idiosincrasia de los conceptos matemáticos, que son difíciles de poner en contextos reales, hace que los estudiantes se aburran en clase, por lo que cuando se aplica una metodología de aula participativa, los alumnos la valoran más en términos de diversión y motivación. Además, los conceptos matemáticos no requieren un aprendizaje tan memorístico como los conceptos científicos, por lo que los alumnos podrían sentirse más motivados a la hora de contestar el cuestionario elaborado en la plataforma Kahoot de contenidos tras la experiencia, ya que podrían obtener mejores resultados (Prieto et al., 2019).

La inmersión apresurada en la virtualidad, agitó los cimientos de la normalidad acostumbrada de la enseñanza. Saliendo a la luz, el esfuerzo agotador por parte de los maestros, quienes utilizaron su creatividad para cumplir sus funciones en un ambiente 
para muchos desconocido (Ardini et al., 2020). Manifestándose, además, el deseo de aprender, expresarse y comunicarse por parte de los y las estudiantes, junto a una manifestación de insuficiencias y desafíos. Es así que muchos docentes que tuvieran años de experiencia en educación presencial se vieron inexpertos ante la nueva realidad educativa: la virtualidad.

La implementación del aprendizaje basado en proyectos en línea en el aprendizaje de las matemáticas es una alternativa que puede ser utilizada para mejorar las habilidades de resolución de problemas creativos de los estudiantes en el aprendizaje con las herramientas virtuales que el docente considere pertinente utilizarlas en su clase (Wahyuningsih et al., 2021). El trabajo mediante proyectos permite que el estudiante se desenvuelva y pueda aprender a trabajar en equipo si se requiere.

La enseñanza a distancia requiere que todos los involucrados maestros y niños tengan acceso a los dispositivos necesarios y puedan utilizarlos de manera adecuada. Para varias familias, esto a menudo significaba que padres e hijos tenían que compartir los dispositivos, ya que todos participaban en una actividad remota algunos para trabajar y otros para estudiar. Obviamente, esto tuvo repercusiones en la enseñanza en clase, en términos de horarios y la forma en que se utilizaron estas herramientas. Además, para evitar los riesgos asociados al uso excesivo, los niños no podían permitirse pasar demasiado tiempo frente a la pantalla (el dispositivo a veces era un teléfono inteligente). También debe tenerse en cuenta que, para los niños de la escuela primaria, a menudo se necesita la intervención de los padres al iniciar sesión en el dispositivo, a menudo se les llama para ayudar a sus hijos en la gestión correcta y segura de la tecnología (A. M. Martín et al., 2021).

\section{CONCLUSIÓN O CONSIDERACIONES FINALES}

Tener una educación a distancia de calidad, en medio de una pandemia, es un gran desafío. A pesar de las diferentes dimensiones tan complejas, su viabilidad es incuestionable.

Los recursos virtuales utilizados en esta nueva realidad educativa deben estar presentes en toda realización de clases de parte de los docentes, permitiendo mejores experiencias de diseño y planificación para los docentes.

El hecho de generar un modelo de educación activa con ayuda de la tecnología, con pizarras interactivas digitales y recursos audiovisuales, generan motivación del estudiante 
al momento de aprender, pues no solo entran en un proceso de adquisición de conocimientos, sino que también se divierten.

\section{Recomendaciones}

Se recomienda a las autoridades educativas responsables que mejoren y desarrollen el aprendizaje digital en la educación matemática durante y después de la era COVID-19 y reevalúen el aprendizaje digital y su importancia en la enseñanza y el aprendizaje de las matemáticas en todos los grados durante y después de la pandemia de COVID-19.

\section{LISTA DE REFERENCIAS}

Bartra, G. (2020). Prácticas telecomunicaciones docentes, modalidad no presencial. PUCP, Ingeniería de telecomunicaciones.

Addimando, L., Leder, D., \& Zudini, V. (2021). Teaching and Learning in the Covid-19 Era: The Experience of an Italian Primary School Class. TOJET: The Turkish Online Journal of Educational Technology, 20(1), 60-67. http://www.tojet.net/volumes/v20i1.pdf\#page=70

Ardini, C., Barrozo, M., \& Corzo, L. (2020). Herramientas digitales de comunicación en contexto COVID 19. El impacto en la relación estudiantes-instituciones educativas en Argentina. Revista ComHumanitas, 11(2), 98-122.

Ayala, R. (2021). Un zoom a la educación virtual:biopolítica y aprendizaje centrado en el estudiante. Elsevier España, 22, 177-180.

Campos, S., Méndez, J., León, Z., \& Napaico, M. (2021). Educación en tiempos de pandemia. una revisión teórica. CiIEG, 1(1), 188-196. doi: https://doi.org/10.26490/uncp.horizonteciencia.2021.21.916

Carpenter, D., \& Joshua, D. (2020). We' re AllTeachers Now: Remote Learning During COVID-19 (pp. 568-594). https://doi.org/10.1080/15582159.2020.1822727

Cedeño-Escobar, M. R., Lucas-Flores, Y. A., Ponce-Aguilar, E. E., \& Peredo-Alonzo, V. E. (2020). Classroom y Google Meet, como herramientas para fortalecer el proceso de enseñanza- aprendizaje. Polo Del Conocimiento, 5(07), 388-405. https://doi.org/10.23857/pc.v5i7.1525

CEPAL-UNESCO. (2020). La educacion en tiempos de la pandemia COVID-19. Comisión Económica Para América Latina y El Caribe, Santiago Oficina Regional de Educación Para América Latina y El Caribe de La Organización de Las Naciones Unidas Para La Educación La Ciencia y La Cultura, 11, 11-13. 
https://unesdoc.unesco.org/ark:/48223/pf0000374075?posInSet=1\&queryId=9cc f4a39-7c50-43e4-856b-a09632daa7a2

Chong, P. G., \& Marcillo, C. E. (2020). Estrategias pedagógicas innovadoras en entornos virtuales de aprendizaje. Revista Científica Dominio de Las Ciencias, 6, 56-77. https://orcid.org/0000-0001-9952-6819

Corrales, J. (2021). Revisión actualizada: Enseñanza de las matemáticas desde los entornos virtuales de aprendizaje. Ciencia y Educación, 5(2), 25-40. https://doi.org/10.22206/cyed.2021.v5i2.pp25-40

Crespo, M. del C., \& Palaguachi, M. C. (2020). Educación con Tecnología en una Pandemia: Breve Análisis. Revista Scientific, 5(17), 292-310. https://doi.org/10.29394/scientific.issn.2542-2987.2020.5.17.16.292-310

Ertmer, P., \& Timothy, N. (1993). Conductismo Cognitivismo y Constructivismo:Una comparación de los aspectos críticos desde la perspectiva del diseño de instrucción. ACADEMIA Accelerating the World's Research., 6(4), 50-72. chrome-

extension://efaidnbmnnnibpcajpcglclefindmkaj/viewer.html?pdfurl=https $\% 3 \mathrm{~A} \%$ 2F\%2Fd1wqtxts 1xzle7.cloudfront.net\%2F33702241\%2F1.-

ConductismoCognositivismo-y-Constructivismo-with-cover-pagev2.pdf\%3FExpires\%3D1627009793\%26Signature\%3DfJEURpqbgYfCWhwLOjD

Francisca, M., Martínez, C., Silvana, G., Alvarez, V., Enrique, C., \& Rodríguez, P. (2021). Student Perceptions of Ecuadorian Covid-19 Pandemic. Creative Commons Attribtion 4.0 International License, 79(2), 242-253. https://doi.org/10.33225/pec/21.79.241

García, Fonseca, \& Concha. (2015). Aprendizaje y rendimiento académico en educación superior: un estudio comparado. Actualidades Investigativas En Educación, 15(3). https://doi.org/10.15517/aie.v15i3.21072

Guzzetti, C. (2020). Plataforma virtual : una herramienta didáctica para el Proceso de Enseñanza Aprendizaje. Revista Multidiciplinar Ciencia Latina, 4(2), 860-877. https://ciencialatina.org/index.php/cienciala/article/view/122/104

Hidayah, I., \& Prayoga, R. A. (2021). Students' attitude towards mathematics in discovery learning using concrete and virtual manipulative. Journal of Physics: 
Recursos virtuales en el aprendizaje...

Conference Series, 1918(4), 1-7. https://doi.org/10.1088/1742$6596 / 1918 / 4 / 042148$

Kraus, G., Formichella, M. M., \& Alderete, M. V. (2019). El uso del Google Classroom como complemento de la capacitación presencial a docentes de nivel primario. Revista Iberoamericana de Tecnología En Educación y Educación En Tecnología, 24(24), 79-90. https://doi.org/10.24215/18509959.24.e09

Latif, M. A., \& Widiaty, I. (2021). Technology implementation to promote digital learning. IOP Conference Series: Materials Science and Engineering, 1098(5), 17. https://doi.org/10.1088/1757-899x/1098/3/032006

Ligon, G. (2021). A Whole New World: Bitmoji Classrooms, Novice Teachers, and Traditionally Marginalized Students' Virtually Learning During a Pandemic. International Management Review, 17(1), 5-11. https://search.ebscohost.com/login.aspx?direct=true \&db=bth\&AN=149660445\& site $=$ ehost-live

Lin, S., Chen, L., \& Kuo, S. (2010). Learning Behaviors and Interaction Patterns Among Students in virtual learning worlds. I-Manager's Journal on School Educational Technology, 6(1), 39-47. https://www.mendeley.com/catalogue/f132754a-ceda34f8-ae3b-

0162f8c1ee36/?utm_source=desktop\&utm_medium=1.19.8\&utm_campaign=op en_catalog\&userDocumentId=\%7B7de2252b-4a19-4df5-8f5ba8c34165f774\%7D

Lovón, M., \& Cisneros, S. (2020). Repercusiones de las clases virtuales en los estudiantes universitarios en el contexto de la cuarentena por COVID- 19: El caso de la PUCP. Propósitos y Representaciones, 8(3), 1-15. https://revistas.usil.edu.pe/index.php/pyr/article/download/588/1086

Marbán, J., Radwan, E., Radwan, A., \& Radwan, W. (2021). Primary and secondary students' usage of digital platforms for mathematics learning during the COVID19 outbreak: The case of the Gaza strip. Mathematics, 9(2), 1-21. https://doi.org/10.3390/math9020110

Marciniak, R., \& Gairín Sallán, J. (2017). Dimensiones de evaluación de calidad de educación virtual: revisión de modelos referentes. RIED. Revista Iberoamericana de Educación a Distancia, 21(1), 217. https://doi.org/10.5944/ried.21.1.16182 
Martín, A. M., Lavandera, S., Mora, B., Sánchez, C., \& Pérez, L. (2021). Working methodology with public universities in peru during the pandemic-continuity of virtual/online teaching and learning. Education Sciences, 11(7), 3-26. https://doi.org/10.3390/educsci11070351

Martín, C. T., Acal, C., Honrani, M. El, \& Estrada, Á. C. M. (2021). Impact on the virtual learning environment due to covid-19. Sustainability (Switzerland), 13(2), 1-16. https://doi.org/10.3390/su13020582

Martínez, Gema. (2017). Tecnologías y nuevas tendencias en educación: Aprender jugando. El caso de Kahoot. Opcion, 33(83), 252-277. https://dialnet.unirioja.es/servlet/articulo? codigo $=6228338$

Martínez, Geraldine. (2020). Recursos y herramientas comunicacionales ante los retos de la educación virtual. Correspondencias \& Análisis, 1(12), 20. https://doi.org/10.24265/cian.2020.n12.10

Molinero, M. del C., \& Chávez, U. (2019). Herramientas tecnológicas en el proceso de enseñanza-aprendizaje en estudiantes de educación superior. Revista Iberoamericana Para La Investigación y El Desarrollo Educativo, 10(0), 5. https://doi.org/10.23913/ride.v10i19.494

Molineros, M. del C., \& Chávez, U. (2019). Herramientas tecnológicas en el proceso de enseñanza-aprendizaje en estudiantes de educación superior. Revista Iberoamericana Para La Investigación y El Desarrollo Educativo, 10, 5. https://doi.org/10.23913/ride.v10i19.494

Morales, J. (2020). Reflexiones sobre la plataforma aprendo en casa del Ministerio de Educación del Perú durante la pandemia covid-19. Unitwin, 6(3), 35-41. https://doi.org/10.35381/cm.v6i3.416

Muñoz-Vázquez, I. G., García-Herrera, D. G., Mena-Clerque, S. E., \& Erazo-Álvarez, J. C. (2020). NEO LMS enseñanza matemática: Uso de recursos digitales. Revista Arbitrada Interdisciplinaria Koinonía, $\quad 5(1), \quad 793$. https://doi.org/10.35381/r.k.v5i1.810

Murillo, F. J., \& Duk, C. (2020). El Covid-19 y las Brechas Educativas. Revista Latinoamericana de Educación Inclusiva, 14(1), 11-13. http://dx.doi.org/10.4067/S0718-73782020000100011

Padrón, C. (2013). Estrategias didácticas basadas en aplicaciones de mensajería 
instantánea Whatsapp exclusivamente para móviles (Mobile Learning) y el uso de la herramienta para promover el aprendizaje colaborativo. Revista de Tecnología de Información y Comunicación En Educación, 7(2), 123-134.

Picón, G. A. (2020). La educación virtual en tiempos de pandemia. Revista de Investigación Científica y Tecnológica, 4(2), 1-3. https://doi.org/10.36003/rev.investig.cient.tecnol.v4n2(2020)prologo

Pincay, V. (2016). Implementación de la plataforma Google Classroom como herramienta de productividad bajo el modelo saas y su aplicación en entornos virtuales de E-A para la autogestión docente como complemento a la modalidad presencial. http://repositorio.ug.edu.ec/bitstream/redug/17722/1/UG-FCMF-BCISC-PTG.1220.pdf

Pratibha, D., Yalagi, P. S., Dixit, R. K., \& Nirgude, M. A. (2021). Effective use of online Teaching-Learning Platform and MOOC for Virtual Learning. Journal of Physics: Conference Series, 1854(1), 1-9. https://doi.org/10.1088/1742$6596 / 1854 / 1 / 012019$

Prieto, C., Palma, O., Tobías, P., \& León, F. (2019). Student assessment of the use of kahoot in the learning process of science and mathematics. Education Sciences, 9(1), 2-13. https://doi.org/10.3390/educsci9010055

Querevalú, P. E., Isaac, G., \& Morales, B. (2021). Estrategias docentes de profesores universitarios en tiempos de Covid-19. Horizonte de La Ciencia, 11(20), 347-360. doi: https://doi.org/10.26490/uncp.horizonteciencia.2021.21.916

Quirós, E. (2009). Recursos didácticos digitales: medios innovadores para el trabajo colaborativo en línea. Revista Electrónica Educare, 13(2), 47-62. https://doi.org/10.15359/ree.13-2.4

Ramitadila, Aliyyah, R., Rachmadtullah, R., Samsudin, A., Syaodih, E., Nurtanto, M., \& Suryanti, R. (2020). The Perceptions of primary school teachers of online learning during the COVID- 19 Pandemic Period: A case study in Indonesia. Of Ethnic and Cultural Studies, 7(2), 90-109. http://dx.doi.org/10.29333/ejecs/388

Rybak, A. (2021). Effectiveness of teaching and learning in technology-supported mathematics education. Journal of Physics: Conference Series, 1946(1), 1-18. https://doi.org/10.1088/1742-6596/1946/1/012004

Sánchez, C. (2020). Herramientas tecnológicas en la enseñanza de las matemáticas 
durante la pandemia COVID-19. Hamut'ay, 7(2), 46-57. file:///C:/Users/San Mateo/Downloads/Dialnet-

LaVirtualidadUnaOportunidadParaInnovarEnEducacion-6584034.pdf

Sánchez, J., Sánchez, P., \& Ramos, F. J. (2012). Usos pedagógicos de Moodle en la docencia universitaria desde la perspectiva de los estudiantes. Revista Iberoamericana de Educación, 60, 15-38. https://doi.org/10.35362/rie600441

Siemens, G. (2004). Una teoría de aprendizaje para la era digital. Academia. Accelerat Ing the World's Research, 1-11.

Silva, M., Paula, M. G., \& Mederos, M. C. (2020). Las TICs y teorías del aprendizaje: Recursos necesarios para la nueva normalidad educativa. Killkana Técnica, 4(3), 1-8. https://doi.org/10.26871/killkanatecnica.v4i3.760

Sosa, E. (2021). Percepciones de los estudiantes sobre la estrategia Aprende en Casa durante la pandemia COVID-19. Academia y Virtualidad, 14(1), 133-150. https://doi.org/10.18359/ravi.5261

Valles-Pereira, R. E., \& Mota-Villegas, D. J. (2020). Kahoot aplicada en la evaluación sumativa en un curso de matemática discreta. Revista Científica, 1(37), 67-77. https://doi.org/10.14483/23448350.15236

Velychko, V. Y., Omelchenko, S. O., Khyzhniak, I. A., \& Fedorenko, E. G. (2021). Developing and using open electronic educational resources in educational activities. Journal of Physics: Conference Series, 1840(1), 1-12. https://doi.org/10.1088/1742-6596/1840/1/012063

Vialart, M. (2020). Estrategias didácticas para la virtualización del proceso enseñanzaaprendizaje en tiempos de COVID-19. 34(3), 1-11. https://orcid.org/0000-0002$1110-4257$

Wahyuningsih, S., Qohar, A., Satyananda, D., \& Atan, N. A. (2021). The Effect of Online Project-Based Learning Application on Mathematics Students' Visual Thinking Continuum in Covid-19 Pandemic. International Journal of Interactive Mobile Technologies, 15(8), 4-17. https://doi.org/10.3991/ijim.v15i08.21565

W. B. e. a. Huitt, "Taxonomy of the cognitive do- main. educational psychology interactive.” Valdosta, Galveston. Valdosta State University, 2011.

Zepeda, G., Salcedo, M., Castañeda, M., \& Fregosa, C. (2017). Implementación de la estrategia

ABP colaborativo. VII Congreso virtual Iberoaméricano de 
Recursos virtuales en el aprendizaje...

calidad en educación virtual

Nayarit,

Tepic.

y a distancia., Universidad Autónoma de

http://www.eduqa.net/eduqa2017/images/ponencias/eje5/5_45_Zepeda_Gabriel-

Salcedo_Monica-Castaneda_Haydee-Fregoso_Carlos-

Implementacion_de_la_Estrategia_ABP_colaborativo.pdf 